\title{
Procedural learning of unstructured categories
}

\author{
Matthew J. Crossley • Nils R. Madsen • F. Gregory Ashby
}

Published online: 11 September 2012

(C) Psychonomic Society, Inc. 2012

\begin{abstract}
Unstructured categories are those in which the stimuli are assigned to each contrasting category randomly, and thus there is no rule- or similarity-based strategy for determining category membership. Intuition suggests that unstructured categories are likely to be learned via explicit memorization that is under the control of declarative memory. In contrast to this prediction, neuroimaging studies of unstructured-category learning have reported task-related activation in the striatum, but typically not in the hippocampusresults that seem more consistent with procedural learning than with a declarative-memory strategy. This article reports the first known behavioral test of whether unstructured-category learning is mediated by explicit strategies or by procedural learning. Our results suggest that the feedback-based learning of unstructured categories is mediated by procedural memory.
\end{abstract}

Keywords Categorization · Procedural learning ·

Memorization

Unstructured categories are those in which the stimuli are assigned to each contrasting category randomly, and thus there is no rule- or similarity-based strategy for determining category membership. An everyday example would be one's personal numbers (e.g., social security number, phone number, locker combination, etc.). Intuition suggests that explicit memorization must be required to learn unstructured categories (Ashby \& O'Brien, 2005), but with feedbackbased training there are some fundamental differences between unstructured-category learning and typical examples of explicit memorization. In standard memorization paradigms, participants are presented with a list of items to memorize. They then study this list until confident that it

M. J. Crossley · N. R. Madsen · F. G. Ashby $(\bowtie)$

Department of Psychological \& Brain Sciences,

University of California,

Santa Barbara, CA 93106, USA

e-mail: ashby@psych.ucsb.edu can be repeated by rote. In unstructured-category learning, the items to be learned are stimulus-category label pairs, and these pairs are not explicitly presented. Rather, they must be inferred from trial-by-trial feedback. Thus, the role of explicit memorization in unstructured-category learning may not be as obvious as it first appears.

The first studies relevant to this issue examined unstructured-category learning using fMRI. The results indicated task-related activity in the striatum, but typically not in the hippocampus or other medial temporal lobe structures (Lopez-Paniagua \& Seger, 2011; Seger \& Cincotta, 2005; Seger, Peterson, Cincotta, LopezPaniagua, \& Anderson, 2010). This pattern of results is consistent with procedural learning, and opposite what one would expect if unstructured-category learning was mediated by explicit memorization (e.g., Papanicolaou et al., 2002; Poldrack et al., 2001). For several reasons, however, these results by themselves cannot be considered diagnostic. First, striatal activation is reported in many different tasks, and second, medial temporal lobe activation is occasionally reported during unstructured-category learning (Seger, Dennison, Lopez-Paniagua, Peterson, \& Roark, 2011) and during other similar tasks, such as the learning of arbitrary stimulus-response associations ${ }^{1}$ (Law et al., 2005). This article reports the first known behavioral test of whether unstructured-category learning is mediated by explicit strategies or by procedural learning.

Procedural memories are the memories of skills that are learned through practice (Willingham, 1998). Traditionally, these have been motor skills, such as those used in athletics

\footnotetext{
${ }^{1}$ It is important to note that hippocampal activation is not, by itself, evidence that declarative memory is active. For example, the hippocampus is also in the default-mode network (Buckner, AndrewsHanna, \& Schacter, 2008), and it is thought to respond to novelty (Strange, Fletcher, Henson, Friston, \& Dolan, 1999), relational encoding (Chua, Schacter, Rand-Giovannetti, \& Sperling, 2007), and stimulus context (Giovanello, Schnyer, \& Verfaellie, 2009).
} 
or in the playing of a musical instrument, but the evidence is now good that certain types of cognitive skills are also learned procedurally. Included in this list are sequences of button presses, such as those learned in the serial reaction time task (Nissen \& Bullemer, 1987), and informationintegration categorization (Ashby, Ell, \& Waldron, 2003; Maddox, Bohil, \& Ing, 2004). In the latter task, accuracy is maximized only if information from two or more noncommensurable stimulus dimensions is integrated at some predecisional stage. Typically, the optimal strategy in such tasks is difficult or impossible to describe verbally (Ashby, Alfonso-Reese, Turken, \& Waldron, 1998).

Several signatures of procedural learning make it qualitatively different from learning that is mediated by declarative memory. First, there typically is little conscious recollection, or even awareness, of the details of procedural memories. Second, procedural learning is slow and incremental, and it requires immediate and consistent feedback ${ }^{2}$ (Willingham, 1998). Third, procedural learning includes a motor component (e.g., Willingham, Nissen, \& Bullemer, 1989). For example, the learning in information-integration tasks is of response positions, ${ }^{3}$ not of abstract category labels. This has been demonstrated in a number of studies showing that switching the locations of the response keys after learning impairs information-integration categorization, but not a form of category learning (i.e., rule-based) that depends on declarative memory (Ashby et al., 2003; Maddox et al., 2004; Maddox, Glass, O’Brien, Filoteo, \& Ashby, 2010; Spiering \& Ashby, 2008). Similar results have been reported for the serial reaction time task (Willingham, Wells, Farrell, \& Stemwedel, 2000).

In this article, we will consider response position learning as a signature of procedural learning. Specifically, after training participants in an unstructured-categorization task, we switched the locations of the response keys (which we will henceforth refer to as a button switch). If unstructured-category learning is mediated by explicit memorization, then previous research suggests that no button-switch interference would occur. However, if unstructured categories are learned procedurally, then switching the response keys should interfere with

\footnotetext{
${ }^{2}$ This second condition is critical, because not all implicit learning is procedural. For example, the perceptual representation memory system (PRS) is thought to mediate perceptual changes that occur simply because of repeated exposure to a stimulus (Schacter, 1990). Behaviorally, one critical difference between PRS-mediated learning and procedural learning is that the former does not require feedback, whereas the latter does. Evidence has suggested that the PRS contributes significantly to A/not-A prototype distortion category learning (Casale \& Ashby, 2008), the learning of statistical regularities in language (Saffran, 2002), and artificial grammar learning (Knowlton \& Squire, 1996).

${ }^{3}$ More recent evidence has suggested that learning in informationintegration tasks might be more generally tied to response features (Spiering \& Ashby, 2008).
}

categorization performance. As a control, we replicated this experiment with rule-based categories that used similar stimuli. In rule-based tasks, the categories can be learned via an explicit hypothesis-testing procedure. In most cases, the rule that maximizes accuracy is easy to describe verbally (Ashby et al., 1998). In the present case, only one stimulus dimension was relevant, and the participant's task was to discover this dimension and then to map the different dimensional values to the relevant categories. Considerable evidence has suggested that rule-based category learning recruits declarative memory (e.g., working memory; see Ashby \& Maddox, 2005, for a review), and as we mentioned above, several studies have shown that switching the response keys after learning does not interfere with rule-based categorization.

Although our main goal was to examine how unstructured categories are learned, a secondary goal was to examine more closely how the use of probabilistic category assignments affects the nature of category learning. A widely held view is that making category assignments probabilistic increases the likelihood of procedural-learning strategies (Knowlton, Squire, \& Gluck, 1994; Knowlton, Mangels, \& Squire, 1996). In contrast to this prediction, Ell and Ashby (2006) reported that the use of probabilistic category assignments in a rule-based task had no effect on the tendency of participants to use strategies (e.g., onedimensional) that appeared to be explicit. Even so, Ell and Ashby's results could not rule out the hypothesis that in the probabilistic conditions, the explicit-appearing strategies had actually been learned procedurally.

This article describes the results of an experiment designed to test whether unstructured-category learning recruits declarative or procedural memory and whether the use of probabilistic category assignments increases the likelihood of procedural learning. To address these questions, we used a $2 \times 2$ factorial design, in which two levels of category structure (unstructured vs. rule-based) were crossed with two levels of category assignment (deterministic vs. probabilistic). All conditions used essentially the same two-category stimuli as in Seger and Cincotta (2005) and immediate trial-by-trial feedback.

\section{Method}

\section{Participants}

We ran 32 participants in the unstructured-probabilistic condition, 20 participants in the unstructured-deterministic condition, 27 participants in the rule-deterministic condition, and 40 participants in the rule-probabilistic condition. All of the participants were undergraduates at the University of California at Santa Barbara with normal or corrected-to- 
normal vision and were given course credit for their participation.

\section{Procedure}

All conditions included one 50-min session consisting of eight blocks of 100 trials each. On each trial, the participant was presented with a fixation cross for $500 \mathrm{~ms}$, followed by a response-terminated stimulus. Immediate auditory feedback was given after each response, with a $730-\mathrm{ms}, 500-\mathrm{Hz}$ pure tone indicating a correct response and a 1,220-ms, $200-\mathrm{Hz}$ tone indicating an incorrect response. On every trial in which the participant emitted a valid response, a 1.5-s intertrial interval followed the stimulus. If the participant took more than $5 \mathrm{~s}$ to respond, he or she was told that the response was too slow, and the trial was discarded. Participants had the option to take a short break at the end of each block of trials.

\section{Instructions}

As in Seger and Cincotta (2005), the participants were given a weather prediction cover story. Specifically, they were told that they were to be presented with a series of line patterns, each one predicting either rain or sun, and that they were to give their responses using one of two designated keys on a keyboard. They were instructed to press the left key (i.e., the "d") with their left hand and the right key (i.e., the " $k$ ") with their right hand. The participants in the two probabilistic conditions were told that the stimuli predicted either rain or sun with reasonable accuracy, but that sometimes the predictions would be wrong. The participants in the two deterministic conditions were told that the stimuli predicted either rain or sun with perfect accuracy. Participants were also notified that there would be a "change" in the experiment near its conclusion, and that they would be given instructions at the appropriate time. The transition between Blocks 7 and 8 was marked with the following message being displayed on the screen: "In the following blocks the two buttons with which you respond have been switched. For every figure to which you responded with 'd' you must now respond to with 'k,' and vice versa. Good luck!'

\section{Stimuli}

For the unstructured-probabilistic condition, we used eight stimuli that were identical to those used by Seger and Cincotta (2005). Specifically, each stimulus was created from five line segments of equal length, including two vertical lines, two lines $15^{\circ}$ below horizontal, and one line $45^{\circ}$ from vertical. Every stimulus contained these same five line segments at the same angles but differed from the other stimuli in how the lines were positioned relative to each other. All eight of these stimuli are illustrated in Fig. 1.
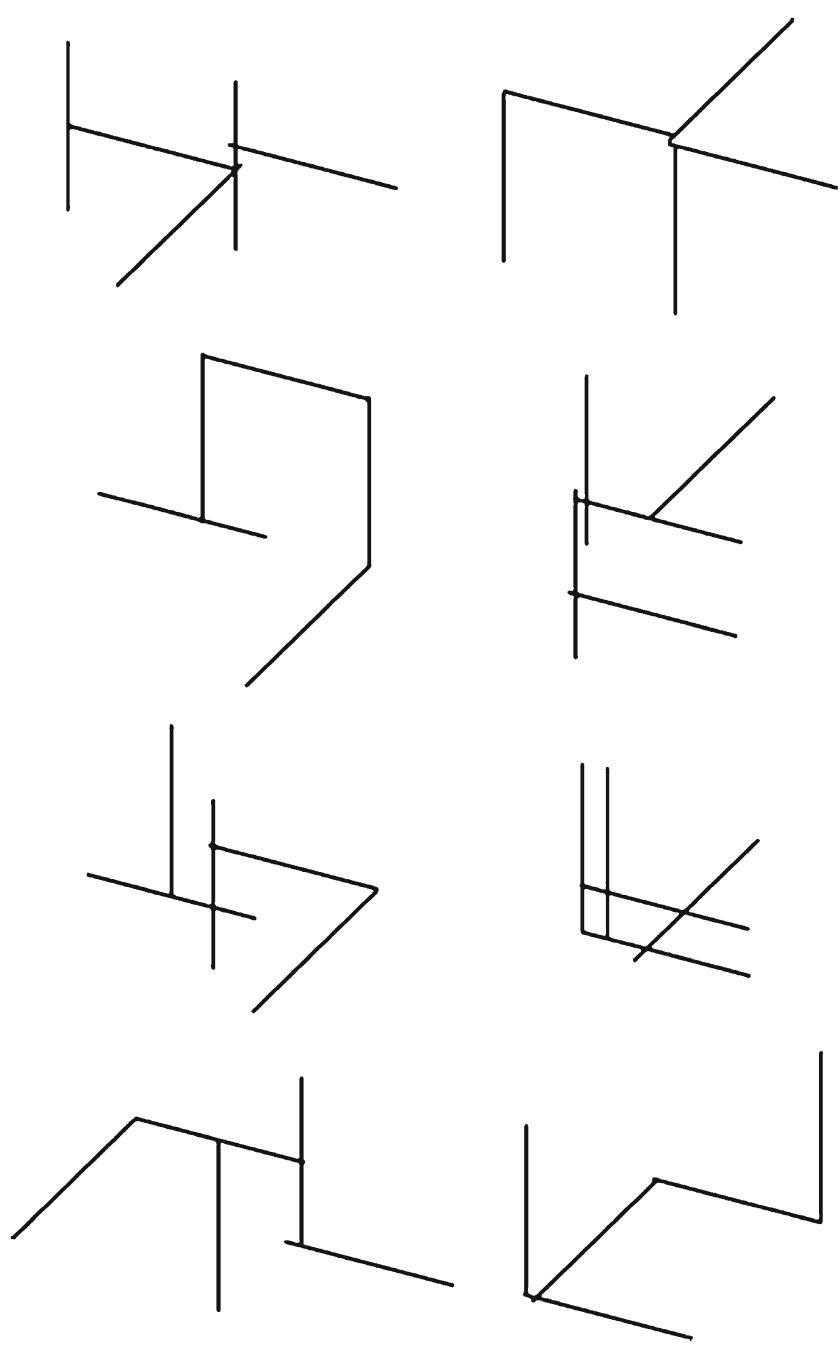

Fig. 1 The eight stimuli used in the unstructured-probabilistic condition

For the unstructured-deterministic condition, we added eight additional line patterns to the eight used in the unstructured-probabilistic condition because with only eight stimuli, the deterministic feedback made the task too easy. The line patterns used in the rule-deterministic and ruleprobabilistic conditions were infused with rule-based structure by coloring one of the lines red and allowing its angle to vary from horizontal to $80^{\circ}$ across stimuli. The angle of this line determined the category membership. Two other line segments were colored blue so that discovery of the relevant dimension was not too easy. The exact algorithm used to generate these stimuli is given in the Appendix, and four example stimuli from these conditions are illustrated in Fig. 2.

\section{Category label assignment}

In the unstructured-probabilistic condition, each stimulus was randomly assigned to either the category "rain" or the 

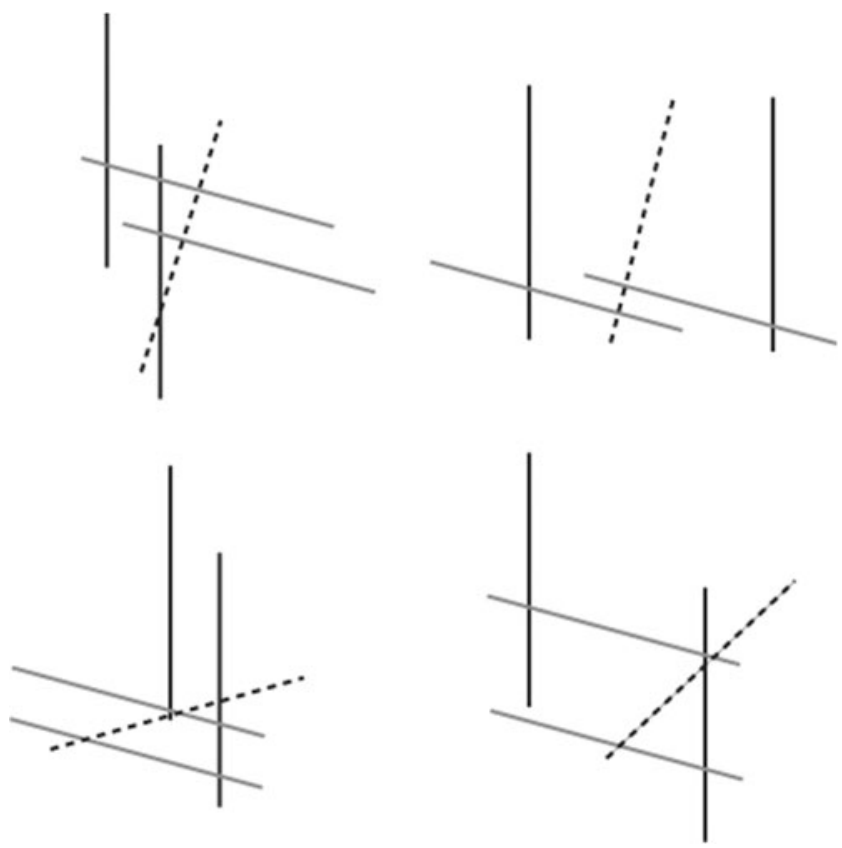

Fig. 2 Example stimuli from the rule-based categories. The exemplars in the top row are in the category "rain," whereas the exemplars in the bottom row are in the category "sun." Solid black lines were presented in black, gray lines were presented in blue, and the broken black lines were presented in red

category "sun," with varying probabilities. Two of the stimuli were given deterministic category assignments; two were assigned to one category $90 \%$ of the time and to the other category $10 \%$ of the time; two were assigned to one category $80 \%$ of the time and to the other category $20 \%$ of the time; and two of the stimuli were assigned to each category $50 \%$ of the time. The assignment of stimuli to categories was randomized for each participant, as was the order of stimulus presentation. These category assignments are exactly as in Seger and Cincotta (2005), although note that our unstructured-probabilistic condition was the union of Seger and Cincotta's probabilistic, deterministic, and random conditions.

In the rule-probabilistic condition, the 800 stimuli were placed into eight bins of roughly 100 stimuli each, depending on the angle of the red line. The stimuli in each bin were assigned to the categories (rain, sun) with the following probabilities: Bin $1\left(0^{\circ}-10^{\circ}\right.$ angle $),(1,0)$; Bin $2\left(11^{\circ}\right.$ $\left.20^{\circ}\right),(.9, .1)$; Bin $3\left(21^{\circ}-30^{\circ}\right),(.8, .2)$; Bins $4\left(31^{\circ}-40^{\circ}\right)$ and $5\left(41^{\circ}-50^{\circ}\right),(.5, .5)$; Bin $6\left(51^{\circ}-60^{\circ}\right),(.2, .8)$; Bin 7 $\left(61^{\circ}-70^{\circ}\right),(.1, .9)$; and Bin $8\left(71^{\circ}-80^{\circ}\right),(0,1)$.

All stimuli in the unstructured-deterministic and ruledeterministic conditions were associated with a given category $100 \%$ of the time. In the rule-deterministic condition, stimuli with a red line sloping more than $40^{\circ}$ above horizontal were assigned to the category "rain," and those with a red line sloping less than $40^{\circ}$ from horizontal were assigned to the category "sun."

\section{Results}

\section{Exclusion criteria}

Button-switch interference, if it exists, can only develop as a result of learning. By definition, a participant who learns nothing during training could not show any effect of a button switch (any interference must interfere with something). Thus, it was important to exclude from the data analysis all participants who failed to learn during training. Unfortunately, of course, the levels of learning of our participants were not binary (i.e., either no learning or perfect learning). As a result, any exclusion criterion based on accuracy was arbitrary. For this reason, we repeated all analyses for four different exclusion criteria. In particular, we excluded participants who did not reach at least $65 \%$, $60 \%, 55 \%$, or $50 \%$ correct during the last training block (i.e., Block 7). The qualitative conclusions of all four sets of these analyses were identical. The results from each analysis are summarized below (in Table 1), but to avoid redundancy, the Results section focuses on the data produced according to the most conservative of these criteria (i.e., excluding all participants who did not reach at least $65 \%$ correct during the final training block). This criterion excluded 11 participants from the rule-deterministic condition, 23 from the rule-probabilistic condition, five from the unstructureddeterministic condition, and 21 from the unstructuredprobabilistic condition.

\section{Accuracy-based results}

Figure 3 shows the mean accuracy across participants (excluding those who failed to reach at least $65 \%$ correct during the final training block) for every 100 -trial block in each of the four experimental conditions. Recall that Blocks 1-7 were training blocks, whereas the location of the response keys was switched in Block 8. Note that in all conditions, accuracy consistently improved during training and then dropped at least a bit in Block 8 (when the buttons were switched). To investigate the effects of the button switch more closely, we computed mean accuracy and response time (RT) differences between Blocks 7 and 8 . Specifically, Fig. 4 shows performance in Block 8 minus the performance in Block 7, so a button-switch deficit is signaled by a positive difference in RTs (i.e., an RT slowing when the buttons are switched) and a negative difference in accuracy (i.e., lower accuracy following the button switch). Note that in both unstructured conditions, the button switch caused accuracy to decrease and RTs to increase, both of which suggest a deficit. In contrast, accuracy dropped slightly in both rule-based conditions, but RTs actually improved. Thus, the performance changes in the rule-based conditions 
Table 1 Statistics ( $t$ values) associated with accuracy and mean RT difference scores between Block 8 (post-button-switch) and Block 7 (prebutton-switch) at various exclusion criteria

\begin{tabular}{|c|c|c|c|c|c|c|c|c|c|c|c|c|}
\hline & \multicolumn{3}{|l|}{$65 \%$} & \multicolumn{3}{|l|}{$60 \%$} & \multicolumn{3}{|l|}{$55 \%$} & \multicolumn{3}{|l|}{$50 \%$} \\
\hline & $\mathrm{N}_{\mathrm{in}} / \mathrm{N}_{\mathrm{ex}}$ & Acc & RT & $\mathrm{N}_{\mathrm{in}} / \mathrm{N}_{\mathrm{ex}}$ & Acc & RT & $\mathrm{N}_{\mathrm{in}} / \mathrm{N}_{\mathrm{ex}}$ & Acc & RT & $\mathrm{N}_{\mathrm{in}} / \mathrm{N}_{\mathrm{ex}}$ & Acc & RT \\
\hline UP & $11 / 21$ & $-3.50 * *$ & 0.10 & $14 / 18$ & $-4.11 * *$ & 0.31 & $16 / 16$ & $-4.29 * *$ & 0.24 & $21 / 11$ & $-3.64 * *$ & 0.97 \\
\hline UD & $15 / 5$ & $-4.39 * *$ & $4.83^{* *}$ & $16 / 4$ & $-3.08 * *$ & $4.94 * *$ & $16 / 4$ & $-3.08^{* * *}$ & $4.94 * *$ & $16 / 4$ & $-3.08^{* *}$ & $4.94 * *$ \\
\hline RD & $15 / 12$ & -1.06 & -2.03 & $16 / 11$ & -1.06 & -1.36 & $16 / 11$ & -1.06 & -1.36 & $18 / 9$ & -1.51 & -0.65 \\
\hline RP & $9 / 31$ & -0.82 & $\begin{array}{l}-1.23 \\
\end{array}$ & $10 / 30$ & -1.00 & $-1.47 *$ & $16 / 24$ & \begin{tabular}{|l|}
-1.44 \\
\end{tabular} & $-2.34 *$ & $21 / 19$ & -1.64 & $-2.13^{*}$ \\
\hline
\end{tabular}

${ }^{*} p<.05 .{ }^{* *} p<.01$. Shaded cells indicate button-switch impairments. Acc, negative $t$ statistics indicate a performance decrement; RT, positive $t$ statistics indicate a performance decrement; $N_{\text {in }}$, number of participants included in the analysis; $N_{\mathrm{ex}}$, number of participants excluded from the analysis

are more consistent with a speed-accuracy trade-off than with a performance deficit.

To test these observations formally, we performed several statistical tests on the accuracy data. First, we performed a 2 (category structure) $\times 2$ (feedback type $) \times 7$ (training blocks) mixed-design repeated measures ANOVA. We found significant main effects of block $[F(6,276)=82.08, p<.001]$, suggesting overall learning with practice, and of feedback type $[F(1,46)=7.10, p<.05]$, which was driven by the better performance with deterministic feedback, relative to probabilistic feedback. The main effect of category structure was not significant $[F(1,46)=0.0, p=.968]$, suggesting no overall difficulty difference between the unstructured and rule-based categories. We also found a significant two-way interaction between block and feedback type $[F(6,276)=6.08, p<.001]$

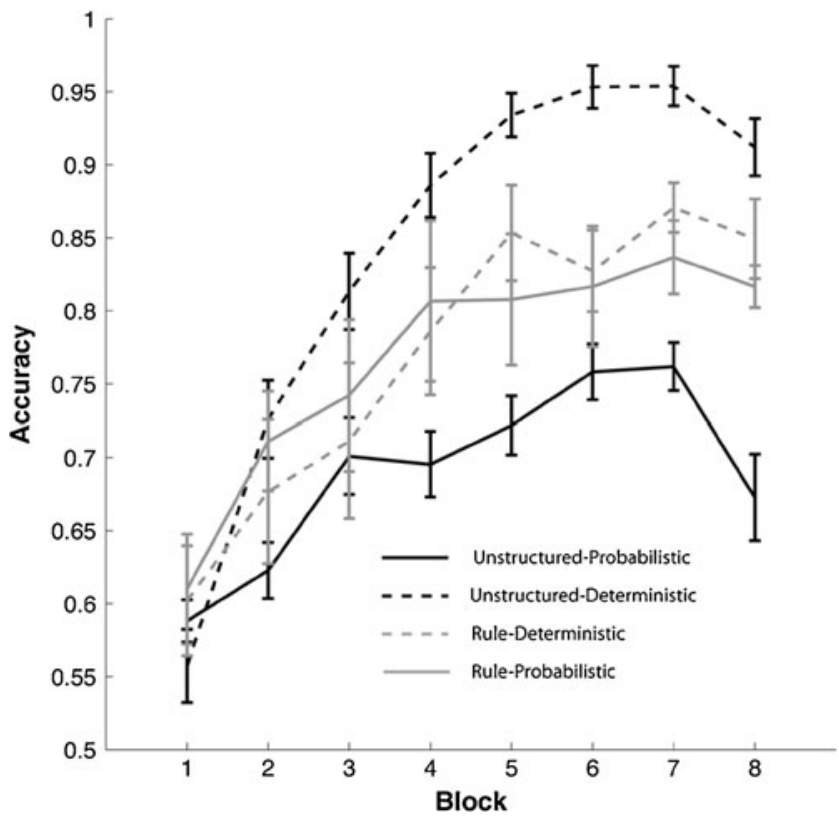

Fig. 3 Mean accuracy in each block of 100 trials across all conditions (excluding participants who did not reach at least $65 \%$ correct by the end of Block 7). Blocks 1-7 were trained with a consistent category label-response key mapping, and these mappings were reversed in Block 8. Error bars represent SEMs that was driven by the faster learning rate with deterministic feedback than with probabilistic feedback. The interaction between category structure and feedback type was also significant $[F(1,46)=7.22, p<.01]$, indicating that deterministic feedback helped unstructured-category learning more than rule-based learning. The block by category structure interaction was not significant $[F(6,276)=1.31, p=.25]$, further confirming that there was no difficulty difference between the rule-based and unstructured categories. Finally, we found a significant three-way interaction among block, category structure, and feedback type $[F(6,276)=3.14, p<.01]$.

To test more precisely whether performance changed when the buttons switched locations, we computed several repeated measures $t$ tests (one per condition) ${ }^{4}$ on the accuracy and RT difference scores between Blocks 7 (last training block) and 8 (transfer block). These $t$ tests revealed that the accuracy drop during the button switch was significant in both the unstructured-probabilistic $[t(10)=-3.50, p<.01$, $d=1.05]$ and the unstructured-deterministic $[t(14)=-4.39$, $p<.001, d=1.13]$ conditions. In contrast, the accuracy change when the buttons switched was not significant in the rule-deterministic $[t(14)=-1.06, p=.31, d=0.24]$ or the rule-probabilistic $[t(8)=-0.82, p=.44, d=0.27]$ conditions. In addition, the button switch caused a significant mean RT impairment in the unstructured-deterministic condition $[t(14)=4.83, p<.001, d=1.25]$, but the pre- and postbutton-switch mean RTs were not significantly different in the unstructured-probabilistic condition $[t(10)=1.82, p=.10, d=$ $0.55]$, the rule-probabilistic condition $[t(8)=-1.23, p=.25, d=$ $0.41]$, or the rule-deterministic condition $[t(14)=-2.03, p=.06$, $d=0.52]$. Thus, these tests confirmed significant button-switch interference in both unstructured conditions, and no interference in either rule-based condition.

\footnotetext{
${ }^{4}$ These $t$ tests were not collapsed across either feedback types or category structures, because an additional 2 (category structure) $\times 2$ (feedback type) $\times 2$ (block: last training block vs. transfer block) mixed-design repeated measures ANOVA indicated significant interactions between category structure and feedback type and between block and category structure.
} 

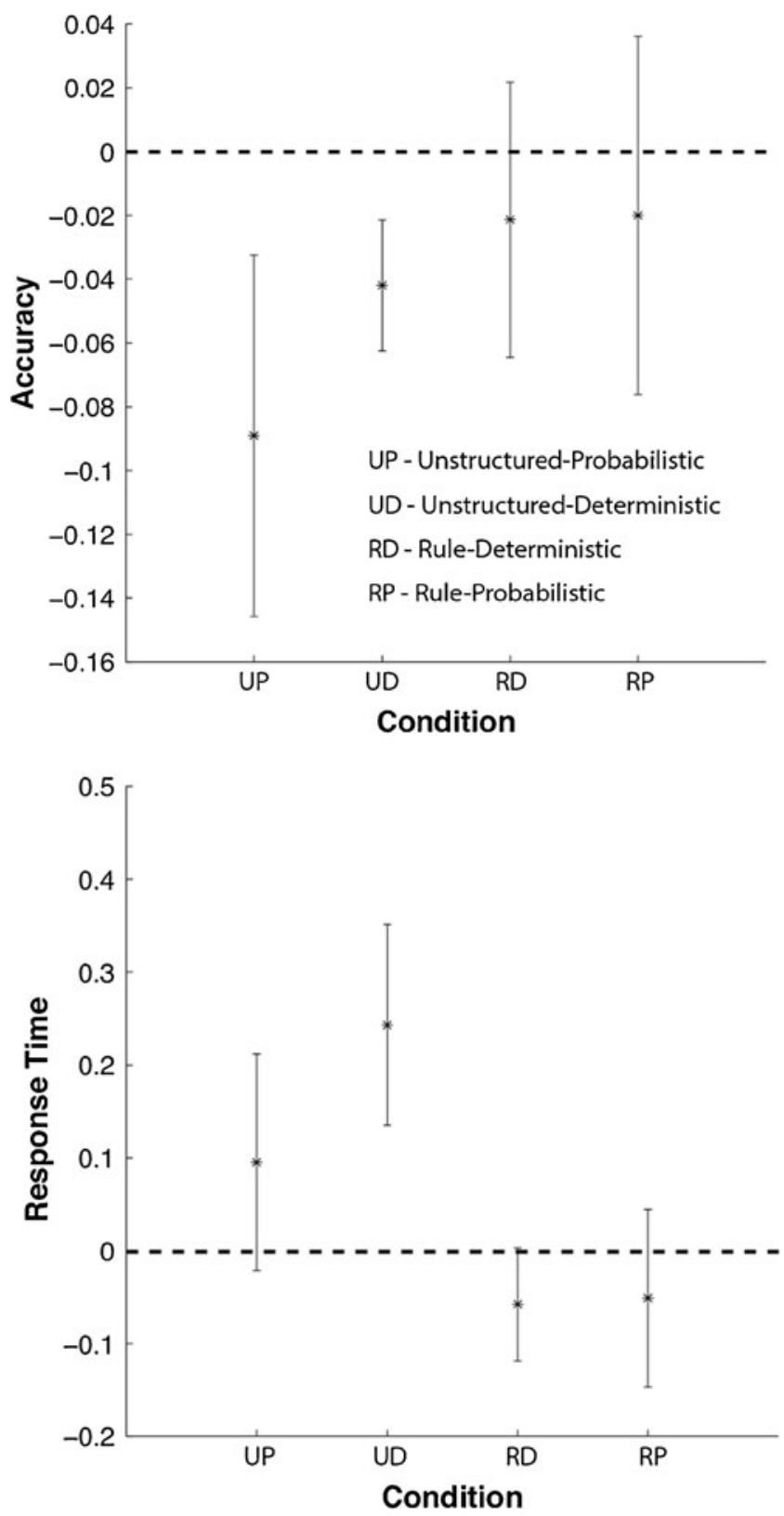

Fig. 4 (Top) Mean accuracy differences between Block 8 (post-button-switch) and Block 7 (pre-button-switch). Note that these figures exclude participants who did not reach at least $65 \%$ correct by the end of Block 7. (Bottom) Mean RT differences between Block 8 (postbutton-switch) and Block 7 (pre-button-switch). Note that negative accuracy difference scores and positive RT difference scores indicate a performance decrement. Error bars represent $95 \%$ confidence intervals

To verify that these results were not due to the arbitrary exclusion criterion that we adopted, we repeated all of the $t$ tests reported here for three other exclusion criteria. The results of these tests are summarized in Table 1, in which the shaded cells indicate significant button-switch impairments. Note that both unstructured conditions exhibit significant impairments under all exclusion criteria, and that neither rule-based condition ever exhibits any significant impairment. Thus, this qualitative feature of our results was invariant over all exclusion criteria.

Finally, we investigated whether the magnitude of the button-switch interference in the unstructured-probabilistic condition varied across the different stimuli. First, before the button switch, accuracy was highest on the two deterministic stimuli and degraded ordinally as category membership became increasingly random [these differences were significant in a one-way, mixed-design repeated measures ANOVA: $F(3$, $62)=81.2, p<.001]$. In fact, the confidence intervals for the mean accuracies of the various stimuli did not even overlap [deterministic labels, $(.841-.916)$; probabilistic labels 90/10, (.711 - .786); probabilistic labels $80 / 20,(.608-.682)$; probabilistic labels $50 / 50,(.462-.537)]$. Second, after the button switch, all accuracy differences among the unstructuredprobabilistic stimuli disappeared $[F(3,83)=0.15, p=.9]$. Thus, in the unstructured-probabilistic condition, the button switch caused participants to respond to all stimuli in a similar manner.

\section{Discussion}

Our results suggest that with unstructured categories, switching the locations of the response buttons causes significant interference in accuracy and either no change or an increase in RTs, whereas with rule-based categories the same button switch causes neither an accuracy drop nor an RT increase. Note that these results did not depend on whether the category assignments were deterministic or probabilistic. Since previous research had suggested that button-switch interference is diagnostic of procedural learning, our results suggest that unstructured categories are learned procedurally. In contrast, making category assignments probabilistic did not seem to change how they were learned. In particular, probabilistic rule-based categories did not show button-switch interference, and thus did not appear to recruit procedural learning.

Note that these results were not driven by task difficultythat is, it was not the case that the button switch only interfered with categorization in the more difficult conditions. An inspection of Fig. 3 clearly shows that the two conditions that showed button-switch interference (i.e., the two unstructured conditions) were at opposite ends of the task difficulty spectrum. Thus, switching the locations of the response keys interfered with the easiest and the most difficult categorization tasks. This implies that our results cannot be accounted for merely by task difficulty.

Our results are consistent with the neuroimaging data of Seger and her colleagues (Lopez-Paniagua \& Seger, 2011; Seger \& Cincotta, 2005; Seger et al., 2010), because they have reported significant task-related activity in the striatum 
but not the hippocampus in a category-learning task that used our unstructured-probabilistic categories, and many previous data have implicated the striatum in procedural learning. On the other hand, our results do not seem consistent with intuitive notions that unstructured categories must require explicit memorization for learning to be successful. One post hoc, and rather speculative resolution of this apparent paradox is that episodic memory surely mediates the participant's conscious recollection of specific stimuli that were shown during the experimental session, but that this type of memory might not be ideally suited for using trial-by-trial feedback to associate a categorization response with each stimulus. Instead, feedbackbased trial-and-error learning is a hallmark of striatalmediated procedural learning.

The striatum is well suited for feedback-mediated trialand-error learning, because the evidence is good that synaptic plasticity at cortical-striatal synapses follows reinforcement learning rules (e.g., Reynolds \& Wickens, 2002) - that is, synapses that were active immediately before positive feedback are strengthened, and synapses that were active immediately before negative feedback are weakened. In contrast, in the hippocampus, a number of theories have proposed that learning follows Hebbian learning rules (e.g., Wallenstein, Eichenbaum, \& Hasselmo, 1998). In other words, all active synapses are strengthened, regardless of the feedback. ${ }^{5}$ Thus, according to this hypothesis, if the category assignments must be inferred from the feedback, as in our experiment, then Hebbian learning would strengthen the incorrect associations that occur on the inevitable error trials as much as it strengthens the correct associations, thereby greatly impairing category learning.

On the other hand, Hebbian learning could mediate category learning if there were no errors-if, for example, the categories were learned via rote rehearsal. This might have been possible with our unstructured categories if we had presented the correct category label alongside each stimulus during training. Participants could then cycle through this list until the stimulus-label pairs were learned. An intriguing prediction is that under these training conditions, the learning would be mediated by explicit memorization, and therefore would not be susceptible to button-switch interference. Note, however, that probabilistic category assignments could defeat this type of memorization strategy, because how can one memorize associations that keep changing? Thus, under some conditions, probabilistic category assignments might reduce the likelihood that participants use declarative memory

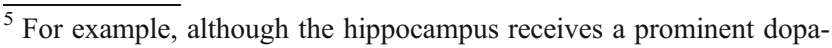
mine projection, unlike the striatum, the hippocampus contains little dopamine active transporter (e.g., Piccini, 2003), which means that hippocampal dopamine lingers in the synapse much longer than striatal dopamine. The poor temporal resolution of the hippocampal dopamine signal makes it an ineffective reinforcement training signal.
}

strategies. Obviously, more work is needed to investigate these interesting questions.

Author note This work was supported in part by Grant No. P01NS044393 from the National Institute of Neurological Disorders and Stroke and by a grant from the U.S. Army Research Office through the Institute for Collaborative Biotechnologies under Grant No. W911NF-07-1-0072. The U.S. Government is authorized to reproduce and distribute reprints for governmental purposes, notwithstanding any copyright annotation thereon. The views and conclusions contained herein are those of the authors and should not be interpreted as necessarily representing the official policies or endorsements, either expressed or implied, of IARPA, DOI, or the U.S. Government.

\section{Appendix: Rule-based stimulus generation}

The rule-based line patterns were composed of one red line that varied in slope from horizontal to $80^{\circ}$ counterclockwise from horizontal (Line 1), two black vertical lines (Lines 2 and 3), and two blue lines sloped $15^{\circ}$ below horizontal (Lines 4 and 5). The following algorithm was used to generate these stimuli:

1. The orientation of Line 1 was chosen randomly (from a uniform $[0,80]$ distribution). The position of Line 1 was centered on the screen.

2. Points on Lines 1 and 4 were randomly selected as the site of their intersection.

3. Points on Lines 1 and 5 were randomly selected as the site of their intersection.

4. Points on Lines 2 and 4 were randomly selected as the site of their intersection.

5. Points on Lines 3 and 5 were randomly selected as the site of their intersection.

A set of 800 stimuli- half to be assigned to the category "rain" and half to the category "sun"-was generated using this method.

\section{References}

Ashby, F. G., Alfonso-Reese, L. A., Turken, A. U., \& Waldron, E. M. (1998). A neuropsychological theory of multiple systems in category learning. Psychological Review, 105, 442-481. doi:10.1037/0033-295X.105.3.442

Ashby, F. G., Ell, S. W., \& Waldron, E. M. (2003). Procedural learning in perceptual categorization. Memory \& Cognition, 31, 11141125. doi: $10.3758 / \mathrm{BF} 03196132$

Ashby, F. G., \& Maddox, W. T. (2005). Human category learning. Annual Review of Psychology, 56, 149-178. doi:10.1146/ annurev.psych.56.091103.070217

Ashby, F. G., \& O'Brien, J. B. (2005). Category learning and multiple memory systems. Trends in Cognitive Sciences, 2, 83-89.

Buckner, R. L., Andrews-Hanna, J. R., \& Schacter, D. L. (2008). The brain's default network: Anatomy, function, and relevance to disease. Annals of the New York Academy of Sciences, 1124, 1-38. 
Casale, M. B., \& Ashby, F. G. (2008). A role for the perceptual representation memory system in category learning. Perception \& Psychophysics, 70, 983-999. doi:s13423-012-0312-0/ PP.70.6.983

Chua, E. F., Schacter, D. L., Rand-Giovannetti, E., \& Sperling, R. A. (2007). Evidence for a specific role of the anterior hippocampal region in successful associative encoding. Hippocampus, 17, 1071-1080.

Ell, S. W., \& Ashby, F. G. (2006). The effects of category overlap on information-integration and rule-based category learning. Perception \& Psychophysics, 68, 1013-1026. doi:s13423-012-0312-0/ BF03193362

Giovanello, K. S., Schnyer, D., \& Verfaellie, M. (2009). Distinct hippocampal regions make unique contributions to relational memory. Hippocampus, 19, 111-117.

Knowlton, B. J., \& Squire, L. R. (1996). Artificial grammar learning depends on implicit acquisition of both abstract and exemplarspecific information. Journal of Experimental Psychology: Learning, Memory, and Cognition, 22, 169-181.

Knowlton, B. J., Squire, L. R., \& Gluck, M. A. (1994). Probabilistic classification learning in amnesia. Learning and Memory, 1, 106-120.

Knowlton, B. J., Mangels, J. A., \& Squire, L. R. (1996). A neostriatal habit learning system in humans. Science, 273, 1399-1402. doi: $10.1126 /$ science. 273.5280 .1399

Law, J. R., Flanery, M. A., Wirth, S., Yanike, M., Smith, A. C., Frank, L. M., \& Stark, C. E. L. (2005). Functional magnetic resonance imaging activity during the gradual acquisition and expression of paired-associate memory. Journal of Neuroscience, 25, 5720 5729. doi:10.1523/JNEUROSCI.4935-04.2005

Lopez-Paniagua, D., \& Seger, C. A. (2011). Interactions within and between corticostriatal loops during component processes of category learning. Journal of Cognitive Neuroscience, 23, 3068-3083.

Maddox, W. T., Bohil, C. J., \& Ing, A. D. (2004). Evidence for a procedural-learning-based system in perceptual category learning. Psychonomic Bulletin \& Review, 11, 945-952.

Maddox, W. T., Glass, B. D., O’Brien, J. B., Filoteo, J. V., \& Ashby, F. G. (2010). Category label and response location shifts in category learning. Psychological Research, 74, 219-236.

Nissen, M. J., \& Bullemer, P. (1987). Attentional requirements of learning: Evidence from performance measures. Cognitive Psychology, 19, 1-32. doi:10.1016/0010-0285(87)90002-8

Papanicolaou, A. C., Simos, P. G., Castillo, E. M., Breier, J. I., Katz, J. S., \& Wright, A. A. (2002). The hippocampus and memory of verbal and pictorial material. Learning \& Memory, 9, 99-104.
Piccini, P. P. (2003). Dopamine transporter: Basic aspects and neuroimaging. Movement Disorders, 18(Suppl. 7), S3-S8.

Poldrack, R. A., Clark, J., Paré-Blagoev, E. J., Shohamy, D., Creso Moyano, J., Myers, C., \& Gluck, M. A. (2001). Interactive memory systems in the human brain. Nature, 414, 546-550. doi: $10.1038 / 35107080$

Reynolds, J., \& Wickens, J. (2002). Dopamine-dependent plasticity of corticostriatal synapses. Neural Networks, 15, 507-521.

Saffran, J. R. (2002). Constraints on statistical language learning. Journal of Memory and Language, 47, 172-196.

Schacter, D. L. (1990). Perceptual representation systems and implicit memory: Toward a resolution of the multiple memory systems debate. Annals of the New York Academy of Sciences, $608,543-571$

Seger, C. A., \& Cincotta, C. M. (2005). The roles of the caudate nucleus in human classification learning. Journal of Neuroscience, 25, 2941-2951. doi:10.1523/JNEUROSCI.3401-04.2005

Seger, C. A., Dennison, C. S., Lopez-Paniagua, D., Peterson, E. J., \& Roark, A. A. (2011). Dissociating hippocampal and basal ganglia contributions to category learning using stimulus novelty and subjective judgments. NeuroImage, 55, 1739-1753.

Seger, C. A., Peterson, E. J., Cincotta, C. M., Lopez-Paniagua, D., \& Anderson, C. W. (2010). Dissociating the contributions of independent corticostriatal systems to visual categorization learning through the use of reinforcement learning modeling and Granger causality modeling. NeuroImage, 50, 644-656.

Spiering, B. J., \& Ashby, F. G. (2008). Response processes in information-integration category learning. Neurobiology of Learning and Memory, 90, 330-338.

Strange, B. A., Fletcher, P. C., Henson, R. N., Friston, K. J., \& Dolan, R. J. (1999). Segregating the functions of human hippocampus. Proceedings of the National Academy of Sciences, 96, 4034-4039.

Wallenstein, G. V., Eichenbaum, H., \& Hasselmo, M. E. (1998). The hippocampus as an associator of discontiguous events. Trends in Neurosciences, 21, 317-323.

Willingham, D. B. (1998). A neuropsychological theory of motor skill learning. Psychological Review, 105, 558-584.

Willingham, D. B., Nissen, M. J., \& Bullemer, P. (1989). On the development of procedural knowledge. Journal of Experimental Psychology: Learning, Memory, and Cognition, 15, 10471060.

Willingham, D. B., Wells, L. A., Farrell, J. M., \& Stemwedel, M. E. (2000). Implicit motor sequence learning is represented in response locations. Memory \& Cognition, 28, 366-375. 